\title{
Formatos escolares alternativos y desigualdades sociales. Resultados de un estudio y reflexiones en torno al derecho a la educación secundaria*
}

\author{
Marcelo Krichesky
}

\section{Resumen}

El presente escrito se propone exponer una aproximación teórica sobre formatos y problemáticas vinculadas con la educación media en la actualidad, para luego trabajar en torno a la indagación realizada hasta el momento en el marco del Proyecto de Investigación "Inclusión educativa en la Argentina 2001-2010 y cambios de formatos de educación secundaria" cuyo trabajo de campo incluye como unidades de indagación a los Centros de Escolarización de Adolescentes y Jóvenes de Buenos Aires, Escuelas secundarias de Reingreso de la Ciudad de Bs As y Bachilleratos Populares. Este trabajo implica una aproximación a la complejidad de estas experiencias organizacionales, como también a las trayectorias y a la subjetividad de adolescentes y jóvenes que acceden a estas propuestas educativas, luego de un proceso de exclusión en la escuela primaria y secundaria.

* Artículo recibido el 28 de julio de 2014. Aceptado el 28 de agosto de 2014.

* Marcelo Krichesky es Licenciado en Ciencias de la Educación - Universidad de Buenos Aires; Magister en Educación y Sociedad - Facultad Latinoamericana de Ciencias Sociales. Doctorando en Educación. Facultad de Filosofía y Letras. Universidad de Buenos Aires marcelo.krichesky@gmail.com 


\section{Palabras clave}

Derecho a la educación - Inclusión - Política educativa.

\section{Abstract}

In this paper we propose a theoretical approach to exhibit on formats and issues related to secondary education. Then we work around the empirical research carried out under the project "Inclusive Education in Argentina 2001 - 2010 format changes and secondary education" which includes field work in different 'School Centers for Adolescents and Youth of Buenos Aires', 'School Reentry of the City of Buenos Aires' and 'Popular Baccalaureate'. It involves an approach to the complexity of these organizational experiences, as well as paths and subjectivity of adolescents and young people entering these educational proposals, after a process of exclusion in primary and secondary school.

\section{Keywords}

Right to education - Inclusion - Educational policy.

\section{Presentación}

En la Argentina posterior a la crisis de 2001, se desarrollaron políticas educativas tendientes a la puesta en marcha y/o consolidación de estrategias de gobierno orientadas a la 'inclusión educativa' y destinadas a que la mayor parte de la población culmine sus estudios secundarios, a instancias del establecimiento de la obligatoriedad que marca la actual Ley de Educación Nacional 26.206 (2006). En este escenario social y político se desarrollan diferentes lineamientos y experiencias promovidas desde el ámbito gubernamental -que flexibilizan el formato escolar tradicional de la educación secundaria- e iniciativas de organizaciones y movimientos sociales orientados a reivindicar el ejercicio del derecho a la educación, especialmente en lo que hace a la finalización de la escolarización secundaria. La multiplicidad de situaciones generadas desde entonces (con heterogeneidad de conflictos, procesos y negociaciones) resulta de interés tanto en la arena gubernamental como también en la sociedad civil en general, dando lugar a la producción de nuevas trayectorias educativas y vitales, como también temas de reflexión y discusión académica.

En este marco, a través del presente artículo se busca recuperar algunos avances realizados durante una investigación en curso desarrollada en la Universidad Pedagógica de la Provincia de Buenos Aires, titulada "Inclusión Educativa en la 
Argentina 2001-2010 y cambios de formatos de educación secundaria. Análisis de políticas públicas de reingreso y bachilleratos populares en Ciudad de Buenos Aires y Provincia de Buenos Aires". ${ }^{1}$ El mencionado proyecto se centra en la preocupación acerca de si los distintos modos de hacer escuela vigentes -tales como los Centros de Escolarización de Adolescentes y Jóvenes (CESAJ) de la Provincia de Buenos Aires, las Escuelas de reingreso de la Ciudad Autónoma de Buenos Aires y los Bachilleratos Populares- inciden en la reconstrucción del lazo social y en procesos filiatorios (Murmis y Feldman, 2002) de adolescentes y jóvenes que forman parte de una capa significativa de la población a quienes se ha vulnerado históricamente el goce de sus derechos sociales y educativos.

Numerosa literatura e investigaciones expresan el problema de la desafiliación social (Castell, 1997; Sennett, 1998) a partir de la crisis o erosión progresiva y acelerada de los dispositivos sociales que aseguran el lazo social. Algunos de los emergentes -sin ser los únicos- de esta crisis lo expresan los adolescentes y jóvenes de los sectores pobres con experiencias de fracaso escolar en el sistema educativo, incorporación -de manera precaria e informal- al mercado de trabajo, y una pérdida de sentido del proyecto vital, es decir una cierta ruptura del lazo entre el presente y el futuro. En el presente artículo nos proponemos generar una reflexión sobre la relación entre nuevos formatos escolares y la recomposición de lazos sociales a partir del cruce a esta frontera escolar. A continuación, nos proponemos mostrar algunos resultados preliminares de la indagación empírica realizada hasta el momento en Centros de Escolarización de Adolescentes y Jóvenes (CESAJ).

\section{Otros formatos en la educación secundaria: las políticas y la investigación educativa}

El análisis sobre las formas escolares abarca una rica producción teórica del campo curricular, la historia y la sociología de la educación. Haciendo una genealogía de la escuela, el formato hegemónico o tradicional se expresó institucionalmente bajo la figura de la "maquinaria" (Varela, Álvarez Uría, 1991; Caruso, Dussel, Pineau, 2001) en términos de tecnología replicable y masiva para aculturar grandes capas de la población, que se desarrolla en la Argentina (como también a nivel global en América Latina y en Europa) con un carácter masivo desde mediados del siglo XIX, logrando articular saberes, tecnologías y relaciones sociales. ${ }^{2}$ Desde hace varias décadas este

\footnotetext{
${ }^{1}$ El proyecto es financiado por la Agencia Nacional de Promoción Científica y Tecnológica (ANPCyT) y la Universidad Pedagógica de la Provincia de Buenos Aires, y se encuentra bajo la Dirección de Marcelo Krichesky. Código de identificación: PICTO 2012-0082. El equipo de investigación está conformado por Maderna, F., Pérez, A., Giangreco, S., Greco, M., Nin, C., Krichesky, M. y Scasso, M.

2 Dichas "tecnologías" "configuran el dispositivo escolar, entendido como un conjunto o una red de elementos heterogéneos, que logra una estabilidad alta en el tiempo". En Caruso, M, Dussel, I, Pineau, P. (2001) se detallan aspectos específicos de dicha maquinaria escolar, como ser el uso del espacio y del
} 
formato escolar resulta un elemento destacado en los diagnósticos y lecturas sobre la crisis educativa, la pérdida del sentido, desfondamiento (Lewkowicz, 2004) y/o declive del programa institucional (Dubet, 2004). Junto a estos aspectos de carácter general se encuentra el debate sobre los cambios de régimen académico, y su incidencia como 'predictor' de trayectorias con menores o mayores niveles de fracaso en el sistema.

El régimen académico, en tanto conjunto de regulaciones sobre la organización de las actividades de los alumnos y sobre las exigencias a las que estos deben responder" (Camilloni, en Baquero Terigi et al. 2009), incluye aspectos como los ritmos y los ordenamientos de las instancias curriculares que deben cursar los alumnos, las instancias de gestión de la convivencia en las que pueden participar, el régimen de evaluación, calificación y promoción, etc. En los últimos años se formularon acuerdos en el Consejo Federal de Cultura y Educación (CFCyE) que promueven políticas de flexibilización del formato escolar. ${ }^{3}$ Los Acuerdos Federales (Resolución Nro. 84/ 09) y decretos de la Provincia de Buenos Aires Nro. 587/2011/ 1480/ 2011) establecen la realización de una serie de modificaciones en normativas y en las prácticas en la búsqueda de recomponer sentidos pedagógicos y con el objetivo de alterar aquellas prácticas anquilosadas o rituales que funcionan como obstáculo para las trayectorias de alumnos y alumnas, ${ }^{4}$ especialmente con relación a las condiciones pedagógicas para que los estudiantes tengan oportunidades reales de cursar con trayectorias no encauzadas (Terigi, 2007).

Asimismo establece la obligatoriedad del apoyo, la orientación y el acompañamiento a los estudiantes en los períodos de diciembre y febrero/marzo como una responsabilidad institucional y bajo la indicación de ofrecer instancias de apoyo en diferentes momentos del año. Por otra parte, los casos de las escuelas medias de reingreso (EMR) de la Ciudad de Buenos Aires (Decreto 408/GCABA/04) y los Centros de Escolarización para adolescentes y jóvenes (CESAJ) de Provincia de Buenos Aires (Resolución 5009/2008 (2008), el programa de inclusión y terminalidad educativa de 14 a 17 años de Córdoba (2010), ${ }^{5}$ entre otros programas de las distintas jurisdicciones del

tiempo, la formación de un cuerpo de especialistas con conocimiento de tecnologías específicas, el docente como ejemplo de conducta, una definición especial de infancia, la conformación de currículas y prácticas universales y uniformes, la descontextualización del contenido académico, la creación de sistemas de acreditación y evaluación

3 La Resolución 84/09 aprueba "Lineamientos políticos y estratégicos de la educación secundaria obligatoria" para dar cumplimiento a la obligatoriedad del nivel. La 93/09 ofrece "Orientaciones para la organización pedagógica e institucional de la educación obligatoria" y la 103/10 aprueba "Propuestas de inclusión y/o regularización de trayectorias escolares".

${ }^{4}$ En el artículo 9 de la Res. CFE No 93 / 09 se promueve la redefinición en un plazo máximo de tres años del régimen académico que regula formas, estrategias, criterios y momentos de la evaluación y la acreditación en el nivel, mediante procesos que garanticen la participación de directivos, docentes y alumnos, y posibiliten la asunción de compromisos y responsabilidades compartidas por parte de los distintos actores implicados.

${ }^{5}$ Las EMR de la CABA, funcionan en ocho establecimientos y tienen un plan de estudios de cuatro años con cuatro niveles de complejidad y un régimen de aprobación por asignaturas cuatrimestral y anual y de 
país, son una expresión de políticas de alta intensidad organizativa y pedagógica, aunque de baja escala (Terigi, Toscano y Briscioli, 2012).

Ciertamente estos casos de experiencias de política pública, si bien resultan una expresión de la limitada capacidad de los sistemas educativos de América Latina para garantizar procesos de alta expansión del sistema (Acosta, 2011), producen innovaciones en lo relativo al modelo organizacional de la escuela secundaria, especialmente en lo que hace a la idea de trayecto, las cargas horarias, la intensificación y la cuatrimestralización de asignaturas y optatividades, la presencia de tutorías y de apoyos escolares, y la incorporación de Formación Profesional (en el caso de CESAJ). No obstante, considerando el universo de jóvenes que abandonan la educación secundaria y la cobertura territorial y de matrícula de estas propuestas que se desarrollan en la Ciudad y en la provincia de Buenos Aires, alcanzarían un porcentaje bajo en relación con la magnitud del abandono que representarían para el caso de la Ciudad (7,7\% del año 2011) y Conurbano en secundaria básica (del 6,8\% en el período $2010-2011),{ }^{6}$ con lo cual la incidencia de estos nuevos formatos en el problema del abandono en la escuela secundaria es de baja escala.

Esta situación de "alta intensidad y baja escala" junto a una baja "ratio", es decir, número de estudiantes por cada profesor, tiene su correlato en un conjunto de propuestas y programas educativos de reingreso y aceleración que se han venido desarrollando en la última década en diferentes Ciudades de América Latina (Montevideo, Bogotá, Medellín, San Salvador, Guatemala, Buenos Aires) en las que se realizaron estudios comparativos (Terigi, 2009). ¿Qué nos dice la investigación educativa de los últimos años?

Los aportes en torno a estos nuevos formatos, en especial con relación a las escuelas de reingreso EMR (Tiramonti, 2011) rescatan las posibilidades inclusivas, con un desarrollo de vínculos que inciden en aspectos emocionales y gratificaciones de la experiencia escolar (Nobile, 2013) y en la reconfiguración del oficio de alumno (Montes y Ziegler, 2010). Entre los elementos claves "para la inclusión", esta investigación recupera, entre otros aspectos, el trabajo docente con ciertos rasgos de militancia, dadas las características vocacionales del trabajo con el otro (Dubet, 2004) centradas en una "misión salvadora" en términos de inclusión en la escuela.

optatividades, con trayectos personalizados y tutorías, de acuerdo a los niveles de los logros. Los CESAJ ofrecen la posibilidad de completar el Ciclo Básico de la educación secundaria, de tres años de duración, en un plazo máximo de dos años, de acuerdo con la trayectoria escolar de cada alumno, respetando el diseño de materias y contenidos centrales previstos en el Diseño Curricular del Ciclo Básico del Nivel Secundario con la inclusión de talleres de formación profesional. Actualmente funcionan 80 CESAJ en la Provincia de Bs As. El PIT 14-17 fue creado por el Ministerio de Educación de la Provincia de Córdoba en 2010. La Resolución Provincial que define su creación ( $N^{\circ}$ 497/10) asume el compromiso de garantizar el acceso y la terminalidad de la escolarización secundaria obligatoria para aquellos jóvenes de entre 14 y 17 años que no hubieren iniciado la escuela secundaria o que la hubieren abandonado de manera transitoria o definitiva. En Córdoba funcionan, a la fecha, 41 centros bajo la modalidad del programa.

${ }^{6}$ DINIECE. Ministerio Nacional de Educacion. Relevamiento anual 2011. 
No obstante, existen alertas acerca del papel que puedan tener estos nuevos formatos de escolarización (cómo resulta esta experiencia de reingreso) en la profundización de la fragmentación educativa y la reproducción de modelos cercados bajo la metáfora (no deseada) del gueto social y cultural. Específicamente, a través de este trabajo encontramos ciertos vacíos que se producen en torno a la "cuestión cultural" en tanto se construye un diálogo que si bien privilegia la exigencia de inclusión,

... sostiene una referencia fuerte en la tarea pedagógica y en el aprendizaje que los jóvenes hacen de los tradicionales saberes escolares, no existe interlocución con las nuevas manifestaciones de la cultura, no hay diálogo de lenguajes, no hay presencia de los nuevos soportes culturales, no hay reconocimiento de los desplazamientos culturales, no hay siquiera una identificación de los jóvenes como subjetividades constituidas en marcos culturales distintos a las referencias escolares. (Tiramonti, 2011: 25)

Otros estudios desarrollados sobre estas ofertas educativas (EMR) (Krichesky, 2008; Krichesky et al., 2011) en diálogo con diferentes actores (directivos, docentes, alumnos) y el que actualmente se está desarrollando en torno a los CESAJ, nos permite pensar que en estos trayectos se "reactualizan" ciertos aspectos de la escuela moderna y del denominado por Dubet como programa institucional. De lo que se estaría hablando es de la presencia de revitalización de procesos de socialización en línea con la construcción de subjetividad y la configuración de procesos de re-filiación social ${ }^{7}$ y proyectos de futuro de mediano plazo. Otro de los efectos sociales significativos se vincula con el reconocimiento del otro y la confianza en él, y con la construcción del oficio del alumno, cuestión que se reitera en el estudio realizado sobre los CESAJ.

\section{Acerca de los CESAJ: características de su régimen académico}

Los Centros de Escolarización Secundaria de Adolescentes y Jóvenes (CESAJ) surgen inicialmente en el año 2008 en la Provincia de Buenos Aires, con el objetivo de generar una propuesta para que los adolescentes y jóvenes de entre 15 y 18 años que se encontraban fuera de la escuela pudieran retomar sus estudios. Según lo establecido en la Resolución N5099/2008 de la Dirección de Educación Secundaria de la Provincia de Buenos Aires, se apela a un formato escolar "que suponga una fuerte revisión de las prácticas de enseñanza, atención a la diversidad y a las particulares condiciones de

\footnotetext{
${ }^{7}$ Acerca del lazo social y la desafiliación, se retoman en este proyecto los aportes de la sociología clásica de Durkheim y Simmel, y los aportes contemporáneos de Castell (1997) que retoma el binomio integración-desafiliación social (Espinoza, 2012) a partir de la crisis del Estado benefactor, la reestructuración económica y sus derivaciones en términos de fragilidad y vulnerabilidad (material y simbólica). Se recuperan en este estudio aportes de la teoría del capital social, y de Elias (2003) la concepción sobre el proceso civilizatorio.
} 
existencia de estos jóvenes" creándose así los CESAJ, diseñados para el reingreso de aquellos estudiantes que en algún momento abandonaron el sistema educativo.

Algunos aspectos del régimen académico se vinculan con los siguientes ítems:

- Carga horaria y estructura curricular. Tienen una carga horaria diaria de 5 horas reloj, 5 veces por semana, lo que significa una carga horaria semanal de 25 horas reloj. Además está contemplada una hora semanal de espacio de tutoría y de formación profesional. En cuanto a la evaluación, ${ }^{8}$ los alumnos no tienen exámenes, sino que deben aprobar cada secuencia didáctica de cada materia para poder iniciar el trabajo con la secuencia siguiente de la misma asignatura. Los alumnos que no aprueban la secuencia en el tiempo previsto continúan trabajando con el docente tutor, a partir de las indicaciones del profesor correspondiente, hasta que se considere oportuno realizar la evaluación que estará a cargo del profesor de la materia.

-Localización. Estos centros pueden funcionar en diversos espacios físicos (una escuela, un espacio de organización social, una parroquia, etc.) pero todos tienen una escuela de referencia, y los alumnos son parte de la matrícula de esa institución. A su vez, los centros están en vínculo directo con una organización social con amplia experiencia comunitaria en el trabajo con jóvenes.

-Docencia y tutoría. El equipo docente es designado por el director a cargo del CESAJ -que es el mismo director de la escuela de referencia- en conjunto con los supervisores y jefes distritales, considerando las características particulares que deben tener los docentes para poder llevar adelante esta experiencia con la población a la que está destinada.

\section{Enfoque y metodología de investigación}

En esta investigación partimos de un enfoque que incluye un estudio de casos analizados con un criterio cualitativo propio de la descripción densa (Geertz, 1987), que contempla elementos propios de la investigación-acción (Carr y Kemmis, 1988), para la cual la interacción y el vínculo con diferentes actores (directivos, docentes y alumnos) aporta lo necesario para comprender mejor la práctica y la reflexión sobre estas propuestas educativas. El análisis en profundidad de estos casos nos permite poner en relación las distintas voces que convergen en la vida escolar, y busca comprender la lógica que organiza la vida social de los actores y de los sentidos atribuidos a sus prácticas en la que puedan combinarse y potenciarse recursos metodológicos diversos,

\footnotetext{
${ }^{8}$ Dirección General de Escuelas (2010) Provincia de Buenos Aires. Disposición de Evaluación N6/10.
} 
pero que apunten a la exploración de los "significados y la comprensión y recrear culturas y contextos del modo evocativo típico de la etnografía" (Woods, 1998, p.).

Este proyecto de investigación integra a) un análisis exhaustivo de fuentes secundarias de información en torno a la escolarización de adolescentes y jóvenes en el Conurbano; b) un estudio de dos casos de CESAJ con directivos, tutores y alumnos; c) la réplica de dicha indagación en una muestra de CESAJ en el Conurbano Bonaerense. A continuación se especifican dichas opciones de indagación. El trabajo de campo se realizó en dos instituciones a partir de 12 entrevistas en profundidad a equipos docentes (10) y directivos (2) y 4 grupos focales con jóvenes de CESAJ. Estas dos instituciones se encuentran ubicadas en los partidos de San Martín (Caso 1) y La Matanza (Caso 2) del Conurbano Bonaerense y ambas registran en la actualidad entre dos y tres cohortes finalizadas de CESAJ. La selección se definió en función del criterio del equipo central de CESAJ de la Dirección de Educación Secundaria de la Provincia de Buenos Aires, al solicitarle experiencias de CESAJ que funcionen en lugares diversos y tengan posibilidad de ser analizadas por un equipo de investigación externo al sistema educativo.

Simultáneamente, se encuestaron en 11 CESAJ del Conurbano Bonaerense (que mantienen continuidad en su gestión desde el 2013) y no se incluyeron los Centros de reciente apertura (2014) encuestando a un total de 100 docentes, 13 tutores y 11 directores. Las encuestas y fichas de relevamiento de trayectorias de los alumnos, se aplicaron en Avellaneda (Deheza), Lanús (Monte Chingolo y Villa Jardín), La Matanza, Berazategui (Hudson y Berazategui), Escobar (Maquinista Savio), Merlo, Quilmes (San Francisco Solano), San Fernando (dos CESAJ), lo que representa el 90\% del total de CESAJ del Conurbano. Del total de CESAJ encuestados (11), solo dos están ubicados en el centro de los partidos, mientras que los nueve restantes se encuentran en barrios cercanos a los centros. En todos los casos, las zonas en las que están enclavados tiene a su alrededor barrios carenciados y/o villas de emergencia. Esta localización tiene un doble efecto paradojal a nivel social: por una parte lograría captar bien la demanda de adolescentes que abandonaron el sistema. Por otra parte, reproduce una dinámica de gueto propio de los procesos de segregación espacial y escolar.

En este estudio se relevó una serie de dimensiones triangulando una diversidad de instrumentos de recolección (encuestas, entrevistas y fichas de alumnos) como ser, entre otras: las condiciones de vida de la población y el clima educativo familiar, las trayectorias escolares previas y la experiencia educativa en el CESAJ, los perfiles y condiciones de trabajo docente, las visiones sobre la propuesta pedagógica, la articulación con el ciclo superior, la perspectiva de los alumnos de su experiencia educativa en CESAJ. Por un problema de extensión de este artículo, las condiciones de trabajo en los CESAJ no son analizadas. No obstante, cabe señalar que en su mayoría son ciertamente desiguales en función de la escuela en la que se localizan, resultando 
más críticas -en cuanto a infraestructura, equipamiento, material didáctico- en aquellos casos que están localizados por fuera de la escuela

\section{La ubicación institucional y territorial de los casos analizados}

La experiencia CESAJ se desarrolla, en todos los casos, asociada con una escuela secundaria de referencia. Esta última es la que se responsabiliza del proceso administrativo de recepción y certificación de los alumnos que recorrieron este trayecto formativo. De acuerdo a la encuesta realizada en 11 CESAJ, más de la mitad (7) de estas propuestas se gestionan en espacios por fuera de la escuela (clubes, sociedad de fomento, ONG, iglesias, Programa Envión). Por la información relevada, los CESAJ que funcionan por fuera de la escuela presentan condiciones institucionales más precarizadas (en infraestructura, equipamiento, articulación con el sistema, etc.) lo cual dificulta que esta experiencia resulte exitosa en términos de reinserción educativa. Estaríamos hablando de condiciones institucionales, que reproducirían ciertos procesos de segmentación y segregación de la oferta del sistema educativo. Asimismo, como se observa en el punto 5, captarían una población adolescente y joven con mayores niveles de vulnerabilidad social.

Los CESAJ indagados en el presente estudio dan cuenta de particularidades por su territorialidad, la articulación con la escuela de origen y las características socioeconómicas y educativas de su población. El Caso 1, ubicado en el Partido de San Martín, se implementa en una Escuela Secundaria Básica a partir de un acuerdo con un Centro de Formación Profesional ubicado en una zona céntrica del Partido. A través de este acuerdo, este CESAJ se desarrolla por la mañana, tres veces por semana con materias curriculares y las otras dos de Formación Profesional. Los varones del CESAJ hacen soldadura y carpintería, y las mujeres, peluquería. Por su parte, el Caso 2 está ubicado en el Partido de La Matanza y se desarrolla en una escuela secundaria que ofrece los seis años, resultando, en este caso, un trayecto formativo de reinserción al ciclo superior de dicha institución. Funciona por la mañana en dos salas ubicadas en una planta baja, en el mismo establecimiento que tiene su secundaria (con ciclo básico y superior) en el primer piso. Este CESAJ ya tiene tres años de antigüedad y sus alumnos, en gran parte, son adolescentes que en su gran mayoría abandonaron dicha escuela.

\section{La exclusión social bajo la lente de los docentes}

La población adolescente y joven que asiste al CESAJ padece condiciones de vida enmarcadas en la pobreza y la vulnerabilidad social. En su mayoría viven en condiciones habitacionales precarias (casillas de chapa o casas de material con techos inadecuados) o en situación de hacinamiento. Un $25 \%$ aproximadamente vive en villas, 
asentamientos y casas ocupadas. Un 77\% vive en casas de material, con menor nivel de precariedad, en barrios obreros con acceso a los servicios básicos. Hay algunos casos de jóvenes que vienen de clases medias pauperizadas, pero representan en esta encuesta menos del 3\%. En línea con los estudios realizados por Auyero (2012) y Pérez Sosto y Romero, (2012: 103-110), dedicados a explorar las condiciones de vida de los jóvenes de 15 a 24 años residentes en el Conurbano bonaerense, se perciben y reiteran ciertas conclusiones respecto de la experiencia vital de estos jóvenes, entre otras cuestiones, los tópicos que emergen de la exclusión social, asociados a la violencia (familiar y/o pasaje por circuitos en vinculación con fuerzas de la policía) que viven cotidianamente, y al consumo de sustancias ilegales.

En el conjunto de problemáticas que presentan los jóvenes que asisten al CESAJ, el consumo de sustancias adictivas resulta un tema recurrente en las entrevistas y encuestas realizadas a docentes, tutores y profesores (Cuadro 1). Parecería que las problemáticas sociales se profundizan más en la población que asiste a los CESAJ localizados por fuera de las instituciones educativas, lo cual daría cuenta, en cierta medida, de que la población con mayor vulnerabilidad elige reincorporarse a espacios educativos no vinculados con la institución social Escuela que años atrás los dejó fuera del sistema.

Cuadro 1- Percepción docente de problemáticas más recurrentes según localización del CESAJ

\begin{tabular}{|c|c|c|c|c|c|c|c|c|}
\hline \multirow{2}{*}{ Problemáticas } & \multicolumn{4}{|c|}{$\begin{array}{l}\text { CESAJ Dentro de la } \\
\text { escuela }\end{array}$} & \multicolumn{4}{|c|}{$\begin{array}{llll}\text { CESAJ } & \text { Fuera } & \text { de } & \text { la } \\
\text { escuela } & & & \end{array}$} \\
\hline & Ito & dio & ajo & $/ R^{N}$ & Ito & dio & ajo & $\mathrm{R}^{\mathrm{N} /}$ \\
\hline $\begin{array}{l}\text { Alumnos cercanos/próximos a } \\
\text { situaciones de conflicto con la ley }\end{array}$ & $2 \%$ & $8 \%$ & $0 \%$ & 2 & $8 \%$ & $\%$ & $2 \%$ & $4 \%$ \\
\hline Consumo de sustancias adictivas & $6 \%$ & $\%$ & ${ }^{2}$ & $\%^{8}$ & $2 \%$ & $\%$ & $\%^{2}$ & $2 \%$ \\
\hline Violencia de género y/o familiar & $8 \%$ & $\%$ & $2 \%$ & $\begin{array}{c}1 \\
2 \%\end{array}$ & $2 \%$ & $\%$ & $6 \%$ & $2 \%$ \\
\hline
\end{tabular}

Fuente: Elaboración propia, encuesta a docentes.

Las trayectorias escolares que tienen los alumnos en su historial previo al ingreso al CESAJ se caracterizan por procesos de repitencia y abandono escolar en la educación primaria y secundaria. Si se considera que en forma acumulada, el $86 \%$ de los estudiantes de CESAJ repitió alguna vez en su itinerario escolar, y experimentó situaciones de abandono escolar, estos datos ponen en evidencia que las trayectorias de estos estudiantes por la educación común básica han estado signadas por experiencias de frustración. Los datos de escolarización de las familias de estos 
adolescentes y jóvenes que asisten a los CESAJ (cerca del 70\% con primaria incompleto y primaria completo) dan cuenta de que el clima educativo familiar de esta población es mayoritariamente adverso. Se define como bajo en aquellos casos en los que el promedio de años de escolarización de los miembros mayores de 17 años residentes en el hogar es inferior a 6 (IIPE, 2008).

Considerando estos datos de contexto, sin duda, esta población que asiste a los CESAJ conformaría para la mayoría de sus grupos familiares, una primera generación que asiste a la escuela secundaria. En los casos estudiados, resulta muy significativo el alto índice de repitencia y abandono en el nivel primario. Las entrevistas realizadas destacan la experiencia previa de lo que significa haber quedado fuera del sistema educativo, la valoración de una oportunidad y una flexibilidad no previstas en los sistemas escolares tradicionales, la ventaja de trabajar en grupos reducidos donde cada uno parece ahora sentirse parte de algo más amplio que los compromete, a la vez que los acompaña. Tal como expresan algunos de los entrevistados, no es menor el desafío que implica, para la gran mayoría de estos/as estudiantes, saber que hay un proyecto que les propone acercarse a algo novedoso para sus familias, como es la obtención de un título de educación secundaria.

\section{La experiencia educativa, re-filiación social y la conformación del oficio de alumno}

Resulta interesante observar ciertos quiebres en la experiencia educativa que viven los jóvenes en los CESAJ en el sentido de adaptarse al ritmo escolar. Ciertamente, en el grupo que logra permanecer en este trayecto formativo (un promedio del $60 \%$ de los que ingresan) hay cambios significativos que son acordes a la configuración del oficio de alumno, ya señalado en anteriores investigaciones (Krichesky, 2011; Montes, 2010). En el Cuadro 2 se observa cómo aumentan los niveles de concentración en la escuela, aspecto muy complejo de sostener al inicio en el CESAJ, y que al segundo año presentan sensibles mejoras. De la misma forma, estas modificaciones sustantivas se observan en aspectos motivacionales para aprender y en los vínculos con los compañeros, los tutores y los docentes. Habría también, aunque en menor medida, cambios en relación con el aprendizaje de contenidos prioritarios, lo cual permitiría dar cuenta de que este proceso de reescolarización facilita un proceso de regreso a la escuela, y a las reglas solicitadas por el sistema, para permanecer y luego egresar.

En el estudio de los casos, se constatan estas tendencias. Parecería que tiene lugar un proceso de metamorfosis o reconfiguración identitaria de los jóvenes durante el trayecto en el CESAJ, y que consolida la llamada 'construcción del oficio de alumno'. Un directivo expresa: "El grupo como entraron ahora no es lo mismo que a principio de año. Costó. Hay una resistencia de parte de ellos para empezar a estudiar. El año pasado fue muy complicado. Y notamos la diferencia". Desde la mirada de los jóvenes 
de los C1 y C2, hay acuerdo respecto a que hay una aceptación de las reglas de construcción del oficio de alumno en relación con tareas y vínculos con pares y docentes. Donde aparece cierta divergencia es en la solidez de la figura de estudiante incorporado a la lógica institucional. En general, los alumnos del C1 tienen temor respecto a su futuro escolar una vez que abandonen el CESAJ. "Nos gustaría seguir juntos y que el CESAJ llegue hasta 6to". En cambio, los jóvenes del C2 están ansiosos por "subir" a la secundaria (la misma funciona en el piso superior del edificio que ocupa el CESAJ). Una posible hipótesis es que al estar la experiencia CESAJ en el mismo colegio, y al compartir profesores, los estudiantes lo ven como un recorrido natural. Cabe agregar que la localización del CESAJ también resulta condicionante de las posibilidades de articulación con el ciclo superior. De la encuesta tomada, se incrementarían las posibilidades de continuidad notablemente en los CESAJ ubicados en la misma escuela.

En este estudio partimos del concepto de experiencia escolar como el conjunto de prácticas y relaciones cotidianas en las que se involucra el sujeto. Dichas prácticas y las relaciones que estas configuran se desarrollan en determinados ámbitos -familiar y escolar- cuya modalidad institucional condiciona el carácter y el sentido que adquiere para otros y para el propio sujeto, como así también las apropiaciones que este realiza. Esta conceptualización permite explicar, simultáneamente, tanto las prácticas materiales, en las que socialmente el sujeto participa, como los procesos de significación que va construyendo. Asimismo, constituye los límites y las posibilidades de cada ámbito institucional en términos de contexto (Achilli, 1999).

Por otra parte, en la investigación realizada se diferencian las trayectorias de carácter teóricas de las reales. Las trayectorias teóricas expresan itinerarios en el sistema que siguen la progresión lineal prevista por este en los tiempos marcados por una periodización estándar" (Terigi, 2009: 2). Contrariamente, las trayectorias escolares reales expresan los modos en que gran parte de los niños y jóvenes transitan su escolarización. Por cierto, "modos heterogéneos, variables y contingentes" (Terigi, 2009: 4) que podemos vislumbrar a través de la combinatoria de algunos datos de la estadística educativa y de los censos nacionales. En general, los estudiantes de los CESAJ poseen edades marcadamente superiores a las que se consideran como 'teóricas' o 'esperables' para el cursado del nivel secundario básico: se concentran entre los 16 y 17 años (edad teórica correspondiente a los dos últimos años de la educación secundaria), lo cual va en directa relación con la población potencial a la que va destinada esta propuesta, según la normativa provincial. Tanto en el C1 como en el C2 resulta muy significativo el alto peso de repitencias y abandono en la educación primaria, lo cual ya da cuenta de los niveles de vulnerabildad de la población que accede a estas instituciones. 
Al acceder a los CESAJ los estudiantes suelen manifestar que en experiencias previas sentían "...que eran ignorados, que no eran escuchados (y que) no han tenido quien los escuche, quien los asesore. (...) Sobre todo los que vienen de escuelas grandes, y otros por cuestiones familiares que han tenido que dejar. Al principio no ajustarse a ninguna norma. El izamiento de la bandera, la gorrita, hablando con el otro, el celular con la música a todo lo que da, en el recreo estar fumando un porro como si fuera lo más normal". Resulta interesante observar ciertos quiebres en estas trayectorias de los jóvenes por el CESAJ. Las entrevistas realizadas a docentes enfatizan, en cambio, un aspecto que proviene del momento y del contexto en el que se encuentran: la experiencia previa de lo que significa haber quedado fuera del sistema educativo, la valoración de una oportunidad y una flexibilidad no prevista en los sistemas escolares tradicionales, la ventaja de trabajar en grupos reducidos donde cada uno parece ahora sentirse parte de algo más amplio que los compromete a la vez que los acompaña.

De la misma forma estas modificaciones sustantivas se observan en aspectos motivacionales para aprender y en los vínculos con los compañeros y los tutores y docentes. Habría también, aunque en menor medida, cambios en relación con el aprendizaje de contenidos prioritarios, lo cual permitiría dar cuenta de que este proceso de reescolarización facilita un proceso de regreso a la escuela, y a las reglas solicitadas por el sistema, para permanecer y luego egresar. Cabe señalar que estas diferencias notorias que se dan en los alumnos entre el 1er y 2do año del CESAJ se darían especialmente en los CESAJ localizados en instituciones educativas. Se podría hipotetizar por lo pronto que la conformación del oficio de alumno resultaría más notoria en estos establecimientos, que los ubicados en instituciones no formales.

Cuadro 2 (a) Percepción de docente y cambios en la experiencia de los jóvenes en el CESAJ

\begin{tabular}{|c|c|c|c|c|c|c|c|c|c|c|}
\hline \multirow[t]{2}{*}{ Ponderación } & \multicolumn{2}{|c|}{$\begin{array}{l}\quad \text { Nivel de } \\
\text { concentrac } \\
\text { en clase }\end{array}$} & \multicolumn{2}{|c|}{$\begin{array}{l}\text { Motivac. } \\
\text { Cursado de } \\
\text { la materia }\end{array}$} & \multicolumn{2}{|c|}{$\begin{array}{c}\text { Relac. } \\
\text { Compañeros }\end{array}$} & \multicolumn{2}{|c|}{$\begin{array}{c}\text { Relac. } \\
\text { Docentes }\end{array}$} & \multicolumn{2}{|c|}{$\begin{array}{c}\text { Aprendizaje } \\
\text { de } \\
\text { contenidos } \\
\text { prioritarios } \\
\text { de la } \\
\text { materia }\end{array}$} \\
\hline & 1 & 2 & 1 & 2 & 1 & 2 & 1 & 2 & 1 & 2 \\
\hline Muy bueno & $3^{9,3}$ & 0 & $\begin{array}{r}2 \\
1,33\end{array}$ & 20 & $\begin{array}{r}1 \\
7,33\end{array}$ & $\begin{array}{r}3 \\
0,67\end{array}$ & $9,3^{2}$ & 44 & 2 & $\begin{array}{r}1 \\
4,67\end{array}$ \\
\hline Bueno & $7^{34,}$ & $4,7^{5}$ & $\begin{array}{r}5 \\
3,33\end{array}$ &, $67^{58}$ & $\begin{array}{r}6 \\
9,33\end{array}$ & $2^{5}$ & $1,3^{6}$ & 0 & $2,7^{6}$ & $\begin{array}{r}7 \\
0,67\end{array}$ \\
\hline Regular & 48 & $7,3^{1}$ & 8 & $\begin{array}{c}14 \\
.67\end{array}$ & $\begin{array}{r}1 \\
3,33\end{array}$ & $\begin{array}{r}1 \\
0,67\end{array}$ & 8 & 4 & $2,7^{2}$ & $\begin{array}{r}1 \\
0,67\end{array}$ \\
\hline Malo & 8 & ${ }^{2}$ & $67^{2}$ & $33^{1,}$ & 0 & 0 & 0 & 0 & $67^{2,}$ & $67^{2}$ \\
\hline$N / R$ & 0 & 5 & 0 & 5 & 0 & 6, & 1 & 5 & 0 & 4 \\
\hline
\end{tabular}




\begin{tabular}{|l|r|r|r|r|r|r|r|r|r|r|} 
& &, 33 & & 33 & & 67 &, 33 & 3 & & \\
\hline Total & 10 & 1 & 1 & 10 & 1 & 1 & 1 & 10 & 1 & 1 \\
& 0 & 00 & 00 & 0 & 00 & 00 & 00 & 0 & 00 & 00 \\
\hline
\end{tabular}

Fuente: Elaboración propia, encuesta a docentes.

En el C2 se dan procesos muy similares en cuanto a las transformaciones producidas entre el momento del ingreso y el momento en que los jóvenes están en el segundo año prontos a egresar e integrarse a un ciclo superior de secundaria.

Por otra parte, resulta significativa la idea generalizada en el imaginario de los docentes y los estudiantes acerca de la continuidad en el ciclo superior. Algunos docentes dan cuenta de este pasaje poniendo en evidencia una clara confianza en que, a pesar de los obstáculos, existen posibilidades de continuidad. De hecho, una docente comenta que tiene estudiantes en el ciclo superior provenientes del CESAJ "tengo alumnos del ciclo superior que han sido alumnos míos en el CESAJ. Los veo muy cómodos. (...) De hecho, los chicos que han dejado de venir del ciclo superior no eran los que venían al CESAJ". En otro caso, el docente considera que, en una escala de 0 a 5 , la posibilidad de que sus estudiantes continúen sus estudios en el ciclo superior es de 3 , y que este aspecto puede incluso mejorarse. Sin dudas esta valoración refleja otra de las posiciones "positivas" en relación con sus estudiantes y sus posibilidades.

En otros casos los docentes se refieren a las expectativas que advierten en los estudiantes respecto de esta continuidad. Algunos de los docentes entrevistados recuerdan que desde el inicio de la experiencia encontraban interés de parte de los alumnos de continuar con el ciclo superior: "...por lo menos tener el título secundario y hacer lo que querían. Hay muchos chicos que vos les preguntás y alguna chica quiere ser maestra jardinera, otro quiere ser contador. Tienen expectativas algunos, otros están medio indecisos". Además, surgen instancias de motivación de parte de los adultos, que en general se orientan a convencer a los estudiantes de que es necesario terminar la secundaria para tener un trabajo digno y que es necesario, por ese mismo motivo, ir adquiriendo ciertas normas que les servirán en un futuro laboral y social. Un docente amplía: "Si van a cursar ciclo superior, este es el momento para ir acostumbrándose a lo que son las normas. Nos guste o no, estas son las normas. Para cambiar el sistema primero tienen que pasar por la escuela. Si no les gusta ya lo cambiarán el día de mañana. Pero si van a atravesar el ciclo superior, y más aún, si van a llegar al ámbito laboral que no es tan permisivo como el escolar, van a encontrar dificultades". El tema de la continuidad en los estudios superiores, si consideramos las percepciones docentes, es una tendencia que parecería más resuelta en los casos de CESAJ localizados al interior de las instituciones del sistema educativo. Por el contrario, en los casos de los CESAJ ubicados en asociaciones civiles, Centros de Formación Profesional, Clubes, etc., los índices de continuidad resultan más bajos 
Cuadro 3- Percepción docente sobre al pasaje al ciclo superior según lugar de funcionamiento del CESAJ.

\begin{tabular}{|l|l|l|l|l|}
\hline \multirow{2}{*}{ \% que pasan al C.S } & \multicolumn{4}{|l|}{ Funcionamiento del CESAJ } \\
\cline { 2 - 5 } & \multicolumn{4}{|c|}{$\begin{array}{c}\text { Fuera } \\
\text { Dentro de la Escuela }\end{array}$} \\
\cline { 2 - 5 } & escuela & \\
\hline Menos del 30\% & 0 & 0,0 & 6 & 13,0 \\
\hline Entre el 30\% y el 50\% & 6 & 27,3 & 19 & 41,3 \\
\hline Entre el 50\% y el 70\% & 14 & 63,6 & 16 & 34,8 \\
\hline Más del 70\% & 2 & 9,1 & 5 & 10,9 \\
\hline & & 100, & & \\
\hline & 22 & 0 & 46 & 100,0 \\
\hline
\end{tabular}

Fuente: Elaboración propia, encuesta a docentes.

\section{La perspectiva de los/las jóvenes}

En la construcción de los relatos que los sujetos hacen a partir de sus vivencias en la escuela, la experiencia se ubica dentro de un universo de significados que aglutina el mundo social y cultural en el que esta experiencia se genera. En los grupos focales realizados en dos CESAJ, algunos estudiantes comentaron haber tenido problemas vinculares con personal de la escuela anterior. Uno de los relatos señala "Yo tuve un problema con mi preceptora, ella era muy discriminatoria conmigo en el colegio. Ya no aguantaba más".

De este modo, se advierte que las causas por las que se acercan al CESAJ son variadas: por influencia de amigos que cursan en la institución, por recomendación de la escuela anterior, por insistencia de algún miembro de la familia, pero siempre en función de una situación problemática atravesada en el vínculo entre las instituciones anteriores y las posibilidades o perfiles de estos estudiantes. A partir de lo indagado en las cuatro instancias de grupos focales realizados con estudiantes de dos CESAJ, podemos advertir que existen similitudes en cuanto a las percepciones que giran en torno a las características de ambas instituciones. Por un lado, se destaca la percepción generalizada de que en los CESAJ existe mayor contención y paciencia frente a dificultades que tengan los estudiantes en términos afectivos, sociales o pedagógicos. Uno de los participantes afirma: "es muy diferente a otros colegios... Está más bueno... te ayudan más (...) los profesores, los preceptores... (...) te dan consejos...". En este sentido, es general escuchar que los estudiantes de ambas instituciones expresen muy buenas opiniones respecto de los referentes institucionales. 
En las percepciones de los jóvenes sobre su propia experiencia educativa se presenta la valoración positiva de estas instituciones en la medida en que "le dieron lugar" o fueron aceptados sin demasiados o ningún cuestionamiento, aceptación que, por otra parte, genera un movimiento subjetivo que subvierte el estigma de haberse sentido rechazados en otras instituciones. Por otra parte, la mayoría de los estudiantes consultados afirma que los docentes faltan menos a clase que lo que ocurre en otras escuelas. No obstante, esta percepción generalizada es cuestionada por algunos estudiantes (esto ocurre en ambas instituciones) cuando hacen referencia a la enseñanza y al aprendizaje: mientras que algunos expresan que aprenden más en el CESAJ al que asisten (en comparación a lo que aprendían en otras escuelas), otros opinan lo contrario. Desde la perspectiva de los estudiantes consultados, ni los tiempos dedicados al estudio o a la elaboración de trabajos prácticos, ni las instancias más flexibles de evaluación, parecerían ser un aspecto significativo y consideran que son elementos inherentes al cambio de formato y al régimen académico.

\section{A modo de conclusiones provisorias}

Estudios realizados sobre estas instituciones educativas y los diferentes actores (directivos, docentes, alumnos) relativizarían, en cierta medida, el declive de la institución (Dubet, 2007) a partir de identificar ciertos efectos sociales que se producen en la experiencia escolar vinculados con la restitución del oficio de alumno, procesos de re-filiación social, y mayor legitimación de la autoridad docente. Por una parte, en la población de sectores sociales vulnerables se observa durante el transcurso, y en la finalización del trayecto formativo altas expectativas de continuidad (al concluir los estudios secundarios) vinculadas a aspiraciones profesionales más identificadas con "las típicas aspiraciones de los sectores medios". Quizá pueda representar un indicador de los efectos positivos que en estos adolescentes ha generado la propuesta de inclusión que les brindó la escuela, lo que nos conduce a pensar que puede llegar a ser relevante explorar -en próximas investigaciones- qué sucede con estas expectativas cuando, una vez que egresaron, se enfrentan con las exigencias y los obstáculos que -en términos de acceso y permanencia- plantean los estudios superiores.

Por otra parte, otro de los efectos sociales significativos se vincula al reconocimiento del otro y a la generación de un sentimiento de confianza. En estas instituciones prima la intencionalidad y el derecho de conocer y/o reconocer (Fraser, 2003) la individualidad de cada alumno, su contexto, sus diversas formas de relacionarse con el conocimiento, sus ritmos, es decir una verdadera personalización en la enseñanza. El discurso de los docentes no restringe la visión de la inteligencia a un grupo social, sino a la confianza, la cual apela a supuestos afirmativos que tienen los equipos docentes sobre las condiciones sociales para el aprendizaje. 
Concordante con este aspecto se encuentra la restitución del oficio de alumno. La percepción de los jóvenes sobre la sobrevivencia en la escuela en tanto "alumnos" remite a la adquisición de valores, a una mejor socialización y a la preparación para el futuro, que incluye la posibilidad de insertarse con éxito en estudios superiores y en el mercado laboral. Esta cuestión permite abordar la construcción de la subjetividad en estos formatos y la conformación de cierto oficio para aprender en la escuela (Perrenoud, 2007), ciertamente perdido u olvidado luego de años de interrupción de su vida escolar o fracasos sucesivos. Otro elemento para considerar en estos estudios sobre nuevos formatos tiene que ver con la modificación del perfil social de la población adolescente y joven que asiste a estas instituciones. Si inicialmente fueron creados para los sectores más vulnerables de la población, algunos indicios de este estudio (que surgen de entrevistas a docentes y tutores) dan cuenta de que en los últimos años a estas ofertas educativas accederían sectores pauperizados (resultado de dinámicas de abandono y necesidad de finalización de los estudios secundarios), pero no necesariamente los sectores más excluidos de la población.

\section{Referencias bibliográficas}

ACOSTA, F. (2011). La educación secundaria en foco: análisis de políticas de inclusión en Argentina, Canadá, Chile y España. Buenos Aires: IIPE.

ACHILLI, A. (1999). Práctica docente y diversidad sociocultural. Rosario: Homosapiens.

CARR, W. y KEMMIS, S. (1988). Barcelona: Martínez Roca Editores.

CARUSO, M., DUSSEL, I. Y PINEAU, P. (2001) La Escuela como máquina de educar. Buenos Aires: Paidós.

CASTEL, R. (1997). La metamorfosis de la cuestión social. Una crónica del salariado. $1^{\text {a }}$ edición, Buenos Aires: Paidós.

DUBET, F. (2004). Texto de la conferencia inaugural del Seminario Internacional sobre "Gobernabilidad de los sistemas educativos en América Latina" organizado por el IIPE/UNESCO en Buenos Aires, 24 y 25 de Noviembre de 2004. Traducido por Emilio Tenti Fanfani.

DUSCHATZKY, S. (1999). La escuela como frontera. Reflexiones sobre la experiencia escolar de jóvenes de sectores populares. Lugar: Paidós.

ELIAS, N. (2003). El proceso de la civilización. Investigaciones sociogenéticas y psicogenéticas. México: FCE.

ESPINOZA, F. (2012). Grietas en el tejido social. Experiencias biográficas de jóvenes montevideanos desde los "lugares" del espacio social. Tesis doctoral. Colegio de México.

FRASER, N. (2006). La justicia social en la era de la política de la identidad: redistribución, reconocimiento y participación, en FRASER, N. y HONNETH, A. ¿Redistribución o reconocimiento?: Un debate político filosófico (pp.) Madrid: Ed. Morata.

GEERTZ, C. (1987). La interpretación de las culturas. Barcelona: Gedisa. 
KRICHESKY, M., MigliavACA A., ALCÁNTARA, A., CABADO, ¿? GRECO, M., MEDELA, P. (2008). Escuelas medias en contextos con vulnerabilidad social. Perspectivas sobre gestión institucional y políticas de inclusión educativa en la Ciudad de Buenos Aires. Informe final. GCBA Ministerio de Educación, Dirección General de Planeamiento, Dirección de Investigación. En prensa.

KRICHESKY, M. (Coord.), CABADO, M., SAGUIER, V., GRECO, M. (2011). Formatos institucionales e inclusión educativa en la zona sur de la Ciudad de Bs As. Dirección de Investigación. Gobierno de la Ciudad de Bs As.

KRICHESKY, M. (Coord.), CABADO, M., SAGUIER, V., GRECO, M. (2011). Jóvenes de contextos vulnerables y su experiencia escolar. En Ministerio de Educación del Gobierno de la Ciudad. Dirección de Investigación y Estadística.

LEWKOWICZ I. (2004). Instituciones perplejas. En Pensar sin Estado. La subjetividad en la era de la fluidez. Buenos Aires: Paidós.

LÓPEZ, N. (Comp.). (2008). SITEAL/Sistema De Información De Tendencias Educativas En América Latina La Escuela y los adolescentes. Informe sobre tendencias educativas. Buenos Aires: IIPE. UNESCO.

MURMIS, M. y FELDMAN, S. (2002). Las ocupaciones informales y sus formas de sociabilidad. Apicultores, albañiles y feriantes. En BECCARIA, L., FELDMAN, S., GONZÁLEZ BOMBAL, I. KESSLER, G. MURMIS, M. y SVAMPA, M. Sociedad y Sociabilidad en la Argentina de los 90 (pp. 173-230). Buenos Aires: Biblos.

NOBILE, M. (2013). Emociones y vínculos de la experiencia escolar. El caso de las escuelas de reingreso de la Ciudad de Buenos Aires. Tesis de doctorado., Buenos Aires: FLACSO.

SENNETT, R. (1998). La corrosión del carácter. Las consecuencias personales del trabajo en el nuevo capitalismo. Barcelona: Anagrama.

TERIGI, F. (2007). Los desafíos que plantean las trayectorias escolares. Paper presentado en el III foro Latinoamericano de Educación "Jóvenes y docentes. La escuela secundaria en el mundo de hoy". Organizado por Fundación Santillana. Buenos Aires.

TERIGI, F. (2009). Las trayectorias escolares. Del problema del individuo al desafío de política educativa. Documento de la Organización de Estados Americanos, Proyecto Hemisférico de OEA. Elaboración de Políticas y Estrategias para la prevención del fracaso escolar. Buenos Aires.

TERIGI, F., TOSCANO, A., BRISCIOLI, B. (2012). La escolarización de adolescentes y jóvenes en los grandes centros urbanos: aportes de tres investigaciones sobre el régimen académico y trayectorias escolares. Second ISA Forum of Sociology. Justicia Social y Democratización. Buenos Aires, Argentina.

TERIGI, F. et al. (2012). Adolescentes y secundaria obligatoria. Centros de Escolarización de Adolescentes y Jóvenes (CESAJ). UNICEF/UNGS: General Sarmiento.

TIRAMONTI, G. (2011). Variaciones sobre la forma escolar. Buenos Aires: FLACSO. 
VARELA, J., ÁLVAREZ URÍA, F. (1991). Arqueología de la escuela. Lugar: Ediciones La Piqueta.

WOODS, P. (1998). Experiencias críticas en la enseñanza y el aprendizaje. Barcelona: Paidós.

\section{Revistas}

MONTES N. Y ZIEGLER S. (2010). Miradas sobre una experiencia de cambio en la escuela secundaria. Nuevos formatos para promover la inclusión educativa. En Revista Mexicana de Investigación Educativa, vol. 15, № 47, octubre.

\section{Referencias electrónicas}

BAQUERO, R., TERIGI, F., TOSCANO, A., BRISCIOLI, B., SBURLATI, S. (2009). Variaciones del Régimen Académico en Escuelas Medias con población vulnerable. Un estudio de casos en el área metropolitana de Buenos Aires. REICE. Revista Iberoamericana sobre Calidad, Eficacia y Cambio en Educación [en línea] 2009, 7. Disponible en:

http://redalyc.uaemex.mx/src/inicio/ArtPdfRed.jsp?iCve $=55114094016$

KRICHESKY, M. (2008). Escuelas medias de Reingreso de la Ciudad de Bs As. Dirección de Investigación. Gobierno de la Ciudad de Bs. As.

http://estatico.buenosaires.gov.ar/areas/educacion/dirinv/publica estadistica/escreingr eso.pdf 